\title{
Antibody-associated Autoimmune Neurological
}

\section{Disease}

James A Varley and Sarosh R Irani

\section{Abstract (200 words)}

Autoimmune neurological diseases are a rapidly expanding group of

potentially treatable syndromes. A number of these conditions are believed to be mediated by autoantibodies and are especially important illnesses as they often respond well to immunotherapies including corticosteroids, plasma exchange, intravenous immunoglobulins, cyclophosphamide and/or rituximab. There are now a number of established distinctive clinical clues to diagnose the diseases, definitive investigations, and increasing experience regarding the relative and beneficial effects of immunotherapies. Awareness of these conditions amongst medical generalists - as well as neurologists and psychiatrists - is crucial as early immunotherapy appears to improve overall outcomes.

In this review, we discuss the most common antibody-associated neurological conditions, focusing on autoimmune myasthenic syndromes and neuromyotonia in the peripheral nervous system, and their central nervous system counterparts including neuromyelitis optica and autoimmune encephalitis. We also briefly describe other related conditions including stiff person syndrome and classical paraneoplastic neurological syndromes. We suggest strategies for identification, differential diagnosis, investigation and treatment of these patients. 
Although individually these conditions are not common, collectively they form a significant group of treatable diseases which frequently present to general medicine and are therefore important for all physicians to promptly recognise.

\section{Keywords 5-10}

Myasthenia Gravis, Autoimmune encephalitis, Encephalitis, Limbic Encephalitis, Neuromyelitis Optica, autoimmune neurological disease, Stiff person syndrome.

\section{Introduction}

There are a range of neurological diseases in which the immune system is thought to be the causative factor. These include multiple sclerosis, post-infectious conditions, some forms of epilepsies and encephalitis. In these conditions, there are data to support effector roles for the innate immune system, and for acquired immunity involving $\mathrm{T}$ cell and B cell populations. More recently, a group of conditions have been described where new pathogenic autoantibodies are being discovered annually. The best described such condition is myasthenia gravis. This finding has led to discovery of a range of other similar autoantibody-mediated conditions - most recently involving the central nervous system. These autoantibodies collectively form a growing armamentarium of diagnostic tests which often prompt effective immunotherapies. In this review we will highlight the key clinical points of the most frequently encountered autoantibody-mediated neurological diseases, discuss their differential diagnosis and outline therapeutic strategies. 


\section{Peripheral neurological autoimmune diseases}

\section{Myasthenia Gravis}

Myasthenia gravis (MG) is caused by antibodies targeting nicotinicacetylcholine receptors (nAChR) on the post-synaptic membrane of the neuromuscular junction (NMJ).

\section{Epidemiology}

MG has an incidence of around 5 per million patient years, and the incidence is rising due to an increased recognition of the condition in the elderly. The sex distribution differs based on age, with females affected three times more than males at younger ages, and a male preponderance (2:1) in older age.

\section{Pathogenesis}

There are a two major causative antibodies in MG which target the nAChR and muscle-specific tyrosine kinase (MuSK) (see fig 1). Antibodies against the nAChR are predominantly $\lg \mathrm{G} 1$ and $\lg \mathrm{G} 3$ subclasses and therefore cause complement activation at the postsynaptic membrane. In addition, these antibodies cause internalisation of the $\mathrm{nAChR}$, reducing available surface expression. These effects decrease the post-synaptic membrane's ability to depolarise, leading to less efficient activation of muscle units and the characteristic fatigueable weakness seen clinically. The other well-recognised antibodies are directed against MuSK, also expressed at the postsynaptic membrane of the NMJ. These are predominantly lgG4 subclass and therefore 
do not activate complement. They are thought to modulate MuSK and prevent it from facilitating clustering of $\mathrm{nAChRs}$, leading again to a malfunctioning NMJ. ${ }^{1}$

\section{Clinical features}

MG typically presents over weeks or months with asymmetrical fatigable weakness in the following muscle groups:

- Ocular - ptosis and diplopia. Ocular symptoms are present in $85 \%$ of patients at presentation and progression to a generalised form of disease occurs in the majority, usually within 2 years.

- Bulbar - dysphagia, dysarthria, dysphonia. Dysfunction of these muscles can lead to aspiration pneumonia

- Limb - proximal and upper limb, more commonly than lower limb.

- Facial, respiratory and neck weakness are also well-recognised

Symptoms are variable throughout the day: typically, better after rest of the affected muscle group and worse after its repeated use.

\section{Investigations}

- AChR antibodies - these are positive in around $85 \%$ of patients with clinical signs of MG

- MuSK antibodies - these are positive in around half of the remaining $15 \%$

- Electromyography (EMG) - repetitive stimulation often demonstrates characteristic 'decrement' of compound muscle action potentials 
(CMAPs). Single fibre recordings can demonstrate 'jitter' which represents inter-potential variation.

- Imaging of the chest - CT/MRI should be carried out in all patients to test for the presence of thymoma (found in $15 \%$ ), especially in older patients. Thymic hyperplasia is seen in a further $20 \%$ (usually younger individuals).

\section{Differential Diagnosis}

- Lambert-Eaton Myasthenic syndrome (LEMS) - an antibody-mediated disease of the presynaptic NMJ often presents with weakness. It can be distinguished clinically from MG by its autonomic features, reduced tendon reflexes, limited early ocular involvement and predilection for the proximal lower limbs. In addition, again by contrast to MG, LEMS often improves after prolonged exercise. It is associated with small cell lung carcinoma in $50 \%$ of cases. Antibodies to voltage-gated calcium channels (VGCC) are detected in 90\%. EMG demonstrates reduced CMAPs, initial decrement and subsequent increment after high frequency repetitive stimulation. Management is to treat an underlying tumour if present, and 3,4-diaminopyrimidine, a presynaptic potassium channel blocker. Some patients require immunosuppression in addition.

- Congenital Myasthenia: These are genetic syndromes caused by mutations in proteins of the NMJ. 
- Amyotrophic lateral sclerosis (ALS): particularly bulbar and limb involvement with asymmetry, and often the combination of upper and lower motor neuron features.

- Primary muscle disease: myopathies can mimic MG but typically present more symmetrically without ocular involvement.

- Mitochondrial disease: overlaps exist, especially due to ocular features. Management

- Assess severity - an important complication of MG is respiratory failure. Longitudinal assessments of forced vital capacity (FVC) are required and if $<1.5 \mathrm{~L}$ should prompt referral to ITU to consider assisted ventilation.

- Avoid exacerbations - a myasthenic 'crisis' can be precipitated by stress, surgery, trauma and infection and by certain medications including D-penicillamine, aminoglycosides, botulinum toxin, macrolides and fluoroquinolones.

- Cholinesterase inhibitors - pyridostigmine is used commonly. It prevents the breakdown of acetylcholine in the NMJ. Doses are titrated to symptomatic benefit and side effects. The latter are due to increased parasympathetic tone and include diarrhoea, sweating, excess salivation, constricted pupils and erectile dysfunction. They can be countered with an antimuscarinic agent (propantheline).

- Immunosuppression - corticosteroids are used as first-line immunosuppression. These can cause a worsening in symptoms over the first two weeks if used at high doses. Typically, after achieving an improvement in symptoms, corticosteroids are weaned to an effective 
minimal dose, in order to reduce steroid-induced complications. Often, a steroid sparing immunosuppressant such as azathioprine, mycofenolate mofetil or methotrexate will be added to help reduce steroid doses: these often take 3-6 months to produce clinical benefits. Plasma exchange and intravenous immunoglobulin are used to achieve a rapid clinical response in more severe situations, often in the case of bulbar or respiratory involvement.

- Thymectomy - should be carried out in all patients with a thymoma, as the tumour can have local pressure effects. Many believe that thymectomy for thymic hyperplasia produces an improvement in weakness, especially in young patients with nAChR antibodies.

\section{Prognosis and follow up}

Aims on follow up should be to achieve remission of symptoms and to monitor and minimize potential treatment side effects. Long term, clinical course is variable and recovery can be hampered by relapses, though the majority achieve symptom free remission with treatment.

\section{Peripheral nerve hyperexcitability (PNH)}

$\mathrm{PNH}$ causes patients to develop continuous, involuntary muscle activity giving painful cramping, fasciculations and impaired muscle relaxation (pseudomyotonia). Weakness tends not to be a feature. $\mathrm{PNH}$ is rare and is also referred to by its eponym, Isaac's syndrome; the more severe forms of PNH are often termed neuromyotonia (NMT). Morvan's syndrome describes NMT in addition to an encephalopathy plus hyperhidrosis, sleep disturbance 
and psychosis. Both of these syndromes have been found to frequently associate with antibodies to the voltage gated-potassium channel complex (VGKCc) and specifically contactin-associated protein-2 (CASPR2). CASPR2antibodies should be requested with either presentation as there is a good response to immunotherapy. There is a frequent tumour association, usually a thymoma, and this should be screened for and treated if present.

\section{Central nervous system antibody-associated diseases}

The central nervous system has traditionally been considered an immune privileged site. In recent times, however, an increasing number of central nervous system diseases have been discovered in which the autoantibodies are likely to be pathogenic. These syndromes often show distinctive clinical features and a good response to immunotherapy. We review the commonest ones below.

\section{Myelitis}

Myelitis is inflammation of the spinal cord. It can affect variable longitudinal lengths and transverse areas of the spinal cord. Myelitis commonly occurs in the context of multiple sclerosis (MS), infectious diseases, and also autoantibody-mediated diseases. Below, we focus on the latter group.

\section{Neuromyelitis Optica}

Neuromyelitis optica (NMO) is relapsing demyelinating disease of the central nervous system typically characterised by severe optic neuritis and 
disabling longitudinally extensive transverse myelitis (LETM). It is often recognized by its eponymous term, Devic's disease. NMO has a strong female predominance $(9: 1)$ and peak age of onset is 39 with an age range involving the very old and young. It was long thought to be a subtype of multiple sclerosis with a more aggressive, disabling disease course. However, in MS most of the disability arises gradually over several years during the progressive phase of the disease. The relapses in MS tend to spontaneously resolve. By contrast in $\mathrm{NMO}$, the disability arises after severe relapses, and, therefore, relapse prevention forms the mainstay of management.

Clinical characteristics that separate NMO and MS are the length of the myelitis (longitudinal, involving the cord over more than 3 vertebral segments), severe or simultaneous bilateral optic neuritis. NMO-spectrum disorders (NMOSD), refer to isolated LETM and isolated optic neuritis. Both of these show a poor (often adverse) response to drugs used to treat MS such as interferon-beta and natalizumab.

The discovery of antibodies to aquaporin-4 (AQP4), a water transport channel present in astrocytic foot processes, recharacterised NMO as a primarily antibody-mediated disorder. AQP4 antibodies are present in roughly $90 \%$ of patients with NMO and in approximately $50 \%$ of NMOSD, with seronegative patients tending to have a monophasic disease course akin to Devic's original description. This is different to MS where despite the presence of CSF oligoclonal bands most descriptions of autoantibodies have been refuted over the years.

Management centers on excluding infectious and metabolic causes and giving pulsed IV methylprednisolone, often with plasma exchange or IVIG 
in acutely affected patients. Long term immunomodulation is given to essentially all patients with AQP4 antibodies as their relapse rate is thought to be high. To reduce steroid requirements, as in MG, steroid sparing agents are frequently used. Indeed, there are good observational retrospective data to suggest roughly $60 \%$ reduction in relapses in patients taking Azathioprine, MMF and Rituximab². There is also a growing importance for symptomatic therapies as neuropathic pain is a major factor influencing quality of life in these patients.

More recently, antibodies to myelin oligodendrocyte glycoprotein (MOG) have been described in patients with seronegative NMOSD and predominantly paediatric acute disseminated encephalomyelitis (ADEM). Patients with MOG-antibodies were more likely to have bilateral optic nerve disease, conus spinal involvement and a monophasic disease course, though relapses are possible.

\section{Autoimmune encephalitis}

Encephalitis is brain parenchymal inflammation and has been classically associated with viral infection, particularly HSV. More recently, autoimmune aetiologies have been discovered to be as common and, in some paediatric cohorts, a more common cause of encephalitis. There are a number of neuronal-surface targeted antibodies associated with autoimmune encephalitis ${ }^{3}$ and only the most common two will be discussed in detail (see table 1 and fig 2), with rarer causes explored in table 2.

\section{Limbic Encephalitis}


In limbic encephalitis (LE) the inflammation is centred on the medial temporal lobes (see fig 3A) and gives a clinical syndrome including amnesia, disorientation and seizures. The most common syndrome is associated with antibodies to LGl1.

Leucine-rich glioma inactivated protein 1 (LGI1) antibody LE

Originally described as antibodies directed at the voltage-gated potassium channels, it has recently been discovered that the commonest specific autoantibody target is LGI1, a protein which complexes with VGKCs in brain tissue. LGI-1 antibodies cause a predominantly non-paraneoplastic LE ( $<5 \%$ rate of tumours) in an equal sex distribution with a median age of onset at 68 years. Patients often present over a few days or weeks with anterograde and retrograde memory impairments, disorientation, change in personality, psychiatric features and a number of seizure semiologies. Investigations reveal hyponatraemia in two thirds, often a normal CSF examination, and about $60 \%$ of MRI scans of the brain reveal medial temporal lobe T2 hyperintensities (fig 3A).

The patients may have typical medical temporal lobe seizures but more distinctively have 'faciobrachial dystonic seizures' (FBDS). These are highfrequency (50-100 a day), brief (<3s) seizures involving the arm and iplislateral hemi-face (see Fig 3B), are usually resistant to anti-epileptics but show a marked reduction with immunotherapy. Most importantly, these FBDS patients have been shown to progress to LE and studies suggest immunotherapies may prevent this progression and the resultant cognitive impairment. 
LE can also be caused in $10 \%$ of cases by autoantibodies against the other established VGKC-complex protein, contactin associated protein-2 (CASPR2).

\section{N-methyl, D-aspartate receptor (NMDAR) - antibody encephalitis}

NMDAR-antibodies are associated with a more diffuse encephalitis, and the associated clinical features are localised throughout the brain.

\section{Epidemiology}

This occurs with an incidence of around 1 per million per year, predominantly affecting females with a peak age of onset at 21 years.

\section{Pathophysiology}

The disease is thought to be caused by NMDAR-antibodies which cross-link and internalise NMDARs in the CNS. There is infiltration of $\mathrm{B}, \mathrm{T}$ and plasma cells in the brain of affected patients, the latter are likely to be responsible for local NMDAR-IgG secretion. In around one-third of cases the disease is associated with an ovarian teratoma which has been shown to express NMDA receptors. This probably leads to a breakdown in immunological tolerance and NMDAR-antibody generation.

\section{Investigations}

- NMDAR-Abs - these are best tested from paired serum and CSF samples. This approach also allows the relative amount of 'intrathecal' antibody synthesis to be determined. 
- EEG - Abnormal in the majority and may be useful to monitor for overt seizure activity. This can show a characteristic 'extreme delta brush' pattern

- Lumbar puncture - Lymphocytosis is observed in two thirds of patients, OCBs are present in around half and often late in disease course.

- MRI Brain - In around $80 \%$ of cases, these are normal. When abnormal, white matter or hippocampal T2 hyperintensities are demonstrated.

- Pelvic MRI - is often used to identify associated ovarian teratomas, especially prevalent in around the 16-35 age group.

\section{Clinical Features}

There is frequently a highly-stereotyped progression in symptoms as the disease progresses (see Table 1). Often, there is a short prodrome with headache, fever and flu-like symptoms, usually followed by neuropsychiatric symptoms a few days later.

- Early (the first 2-3 weeks): Initially a psychiatric presentation with delusions, agitation anxiety, mood lability, aggression and hallucinations with more neurological features such as higher cognitive dysfunction including dysphasia and seizures.

- Late (typically after week 3): Complex hyperkinetic movement disorder with autonomic dysfunction and progression to coma in severe cases. The movement can be catatonic or Parkinsonian. 
There is heterogeneity in presentation of the psychiatric syndrome and the movement disorder, and many patients do not require intensive care support.

\section{Management}

The disease typically recovers slowly, over several months with immunotherapy and supportive measures. Early diagnosis and intervention with immunotherapy are crucial as they positively influence recovery and survival. First line treatment is pulsed IV methylprednisolone, with or without plasma exchange and IVIG. 50\% of patients respond to first line therapies. Second line treatments with either rituximab or cyclophosphamide induce a positive response in the majority of those who did not respond to first line treatment (see fig 4). In addition, removal of ovarian teratomas has been shown to improve outcomes. Symptomatic medications to help control the movement disorder, agitation and psychosis are all useful adjuncts in the care of these patients.

\section{Prognosis}

A holistic approach with intensive neuro-rehabilitation focusing on both cognitive and physical domains is crucial. Many patients return to near their baseline function but there is a $10 \%$ mortality with approximately $10 \%$ relapse rate. ${ }^{4}$ The relapses appear to be preventable with immunotherapy.

\section{Glutamic Acid Decarboxylase (GAD)}


GAD is an intracellular enzyme which catalyses a key step in the production of gamma aminobutyric acid (GABA), a major inhibitory neurotransmitter in the CNS. Antibodies to GAD are associated with type 1 diabetes (at low concentrations) and higher concentrations associate with in autoimmune LE and stiff-person syndrome (SPS).

In contrast to LGI1, LE associated with GAD-antibodies is a disease of younger women with a longer symptom duration, poor recovery and limited response to immunotherapy.

\section{SPS}

Stiff person syndrome is a difficult to diagnose clinical entity characterised by insidious paraspinal or lower limb rigidity which develops to affect the abdomen, upper limbs and face. There are superadded muscle spasms typically with a startle response to sound and touch. The pathophysiology of SPS is thought to be secondary to antibody-mediated impairment of GABAergic interneurons in the brain and spinal cord, initiating aberrant muscular contraction. It causes progressive disability with the majority of patients eventually requiring an ambulatory aid. Management is with GABA-augmentation, using either benzodiazepines or baclofen, often in conjunction with immunotherapy, typically IVIG. ${ }^{5}$

\section{Paraneoplastic Antibody Mediated Neurological Diseases}

In the article above we have discussed a number of antibodies directed against neuronal surface expressed antigens which can frequently be 
associated with cancer (including autoantibodies to the NMDAR, VGCC; see table 2 for information on AMPAR and GABAB-antibodies) and respond well to immunotherapy alongside appropriate oncological treatment. There are another group of antibodies, termed the onconeuronal antibodies which are targeted towards intracellular antigens and are almost always associated with an underlying cancer. In general, these conditions are not immunotherapy responsive, with a predominant $\mathrm{T}$-cell driven immune response and a poor prognosis. Treatment centers on controlling symptoms. The main antigens and their common syndromes include:

- $\mathrm{Hu}$ - Encephalomyelitis and sensory peripheral neuropathy. Most often seen with SCLC.

- Yo - Cerebellar syndrome, associated with breast and ovarian cancer.

- $\mathrm{Ri}$ - Cerebellar syndrome, opsoclonus, associated with ovarian and endometrial cancer.

- CV2 - Encephalomyelitis with sensory peripheral neuropathy, associated with thymoma and SCLC.

- Ma2 - LE, SPS and cerebellar syndrome, associated with testicular tumours and is the one 'onconeural' antibody syndrome which shows a response to immunotherapies.

\section{Summary}

Autoantibody-associated neurological diseases are a growing group of conditions with distinctive clinical features, characteristic tumour associations and available diagnostic tests. These tests should be considered promptly in the appropriate clinical scenario to ensure early treatment of patients. 


\section{$\underline{\text { Key Points }}$}

- Autoimmune neurological diseases are more common than previously thought

- They have specific, patterned clinical phenotypes affecting the peripheral and central nervous systems

- A subacute onset (over 2-6 weeks) is common to most autoimmune neurological diseases

- Many have specific antibody tests that are highly sensitive and specific

- Early treatment improves symptoms, improves recovery and decreases mortality, therefore prompt referral and diagnosis is crucial.

Fig 1 - Antigenic targets and key proteins at the neuromuscular junction (NMJ). The key components include the nicotinic acetylcholine receptors (nAChR) which require rapsyn for clustering. Another membrane associated complex also plays a key role in receptor clustering and includes muscle specific kinase (MuSK) which is another autoantibody target in myasthenia gravis. MuSK associates with agrin and low density lipiprotein receptor-related protein-4 (LRP-4).

Fig 2 - The commonest extracellular and intracellular autoantibody targets associated with central nervous system diseases. Shapes are based on 3D crystal structure of protein. These include the NMDAR (N-methyl-D-aspartate 
receptor), AMPAR (a-amino-3-hydroxy-5-methylisoxazole-4-propionic acid receptor), LGl1 (leucine-rich glioma-inactivated 1), CASPR2 (contactinassociated protein 2), GAD (glutamic acid decarboxylase), GABA $A_{A}$ or $G A B A_{B}$ receptors (gamma-aminobutyric acid), GlyR (glycine receptor), DPPX (dipeptidyl-peptidase-like protein-6) and IgLON5 (Ig-like Domain-containing protein-5).

Fig 3 - Features of limbic encephalitis. A) A T2 weighted MRI scan showing characteristic high signal in the medial temporal lobes (arrows) associated with limbic encephalitis. B) A patient having a faciobrachial dystonic seizure, with brief left-sided facial and ipsilateral upper limb dystonic episodes.

Fig 4 - Outcome data after NMDAR-antibody encephalitis. 24 month follow up data of_>500 patients with NMDAR-antibody encephalitis. $97 \%$ of patients treated with first line treatment and responded in the first three weeks went on to have a 'good response' with mRS of 0-2. This number clearly falls if response is not good and if no second line therapy is given. Adapted from Titulaer et al $2013^{4}$.

Table 1 - List of antigens and clinical features with particular reference to central nervous system diseases. Abbreviations used: LE - Limbic encephalitis, FBDS - faciobrachial dystonic seizures, SPS - Stiff Person Syndrome, CS - corticosteroids, PLEX - plasma exchange, IVIG - 
intravenous immunoglobulin, CYP - cyclophosphamide, BDZ benzodiazepines.

Table 2 - List of rarer antibody-mediated encephalitides with key clinical features. LE - Limbic encephalitis, SCLC - Small Cell Lung Cancer, PERM progressive encephalopathy with rigidity and myoclonus. ?ANTIGENS???

\section{Key references}

1. Binks S, Vincent A, Palace J. Myasthenia gravis: a clinical-immunological update. Journal of neurology 2015. Epub ahead of print.

2. Trebst C, Jarius S, Berthele A, et al. Update on the diagnosis and treatment of neuromyelitis optica: recommendations of the Neuromyelitis Optica Study Group (NEMOS). Journal of neurology 2014;261:1-16.

3. Varley J, Vincent A, Irani SR. Clinical and experimental studies of potentially pathogenic brain-directed autoantibodies: current knowledge and future directions. Journal of neurology 2014; 262(4):1081-95

4. Titulaer MJ, McCracken L, Gabilondo I, et al. Treatment and prognostic factors for long-term outcome in patients with anti-NMDA receptor encephalitis: an observational cohort study. The Lancet Neurology 2013;12:157-165.

5. Saiz A, Blanco Y, Sabater L, et al. Spectrum of neurological syndromes associated with glutamic acid decarboxylase antibodies: diagnostic clues for this association. Brain : a journal of neurology 2008;131:2553-2563.

\section{Further reading}


1. Hoftberger R, Rosenfeld MR, Dalmau J. Update on neurological paraneoplastic syndromes. Current opinion in oncology 2015;27:489495.

2. Irani SR, Alexander S, Waters $P$, et al. Antibodies to Kv1 potassium channel-complex proteins leucine-rich, glioma inactivated 1 protein and contactin-associated protein-2 in limbic encephalitis, Morvan's syndrome and acquired neuromyotonia. Brain : a journal of neurology 2010;133:2734-2748.

3. Irani SR, Michell AW, Lang B, et al. Faciobrachial dystonic seizures precede Lgi1 antibody limbic encephalitis. Annals of neurology $2011 ; 69: 892-900$

4. Irani SR, Gelfand JM, Al-Diwani A, Vincent A. Cell-surface central nervous system autoantibodies: Clinical relevance and emerging paradigms. Annals of neurology 2014;76:168-184. 